\title{
The quark propagator at finite temperature
}

\author{
G. Boyd ${ }^{\mathrm{a}}$, Sourendu Gupta ${ }^{1, \mathrm{~b}}$ and F. Karsch ${ }^{\mathrm{a}, \mathrm{c}}$ \\ ${ }^{a}$ HLRZ, c / o KFA Jülich, D-5170 Jülich, Germany \\ ${ }^{b}$ Theory Division, CERN, CH-1211, Geneva 23, Switzerland \\ ${ }^{c}$ Fakultät für Physik, Universität Bielefeld, D-4800 Bielefeld 1, Germany
}

Received 1 June 1992

Accepted for publication 22 July 1992

\begin{abstract}
We study the quark propagator at finite temperature on euclidean lattices in the Landau gauge, and compare the results to an $\mathrm{O}\left(g^{2}\right)$ lattice weak coupling calculation. The screening mass obtained from spatial correlation functions in the chiral symmetric phase is close to the Matsubara frequency. The temporal correlation functions yield a much smaller screening mass, which approaches the perturbative result, $m_{\mathrm{cff}}^{2}=g^{2} T^{2} / 6$, for $T \geqslant 1.75 T_{\mathrm{c}}$. Deviations from the perturbative behaviour are seen for $T_{\mathrm{c}} \leqslant T \leqslant 1.75 T_{\mathrm{c}}$. For $T \leqslant T_{\mathrm{c}}$, the screening masses from both spatial and temporal correlation functions are large and close to half the mass of the $\rho$-meson. Dispersion relations do not show any significant deviations from free particle behaviour.
\end{abstract}

\section{Introduction}

A central issue in the discussion of the finite-temperature chiral symmetry restoring phase transition in QCD is the exploration of the nature of the high-temperature quark-gluon plasma phase. To what extent can it be viewed as an asymptotically free plasma of quarks and gluons and what is the nature of the interactions among these partons in the plasma phase? It is by no means obvious that the fundamental degrees of freedom in the high-temperature phase are those of massless quarks and gluons [1]. The existence of a non-vanishing string tension for spatial Wilson loops [2] in the plasma phase as well as the non-perturbative effects visible in the equation of state above $T_{\mathrm{c}}$ [3] reflect the non-trivial structure of the plasma phase, which has been attributed to the complicated magnetic sector of QCD.

In particular, the Monte Carlo data on spatial hadronic correlators on euclidean finite-temperature lattices [4-6] and the so-called spatial wave functions for mesons [7] lead to a renewed intensive discussion on the nature of the fundamental excitations in the plasma phase. On the lattice, mesonic and baryonic correlators have been studied in different quantum number channels [4-6]. The spatial fall-off

\footnotetext{
1 Present address: HLRZ, c/o KFA Jülich, D-5170 Jülich, Germany.
} 
of the (pseudo-) vector and baryon correlators is governed by the Matsubara frequency, i.e. the screening masses are close to $2 \pi T$ and $3 \pi T$, respectively [6]. The same holds true for the spatial screening length of the quark propagator, which has been analyzed with Wilson fermions in quenched QCD [8]. While this suggests that quarks propagate freely in these channels, there are large deviations from free field behaviour visible in the (pseudo-) scalar channel as well as for mesonic "wave functions" calculated from spatial correlation functions [7].

How far can these observations shed light on the existence of bound states in the QCD plasma phase, with quantum numbers of ordinary hadrons? An answer to this question requires a better understanding of the structure of the euclidean correlators at finite temperature. In particular, we have to learn more about the temporal correlation functions in euclidean time, which, after analytic continuation, give direct information about the poles of the real time propagators $[9,10]$. Do they show free field behaviour in the QCD plasma phase?

In this paper we concentrate on an analysis of the quark propagator at finite temperature, which is the basic ingredient in the construction of all the hadronic correlators studied so far. We study the spatial as well as temporal quark correlation functions. From lattice simulations of QCD with four flavours of light staggered fermions we extract the corresponding screening masses at zero and non-vanishing quark momentum and compare the results with perturbative calculations, both on finite lattices and in the continuum [11-13]. Our main results are:

(i) The exponential decay of the correlation functions is significantly different in temporal and spatial directions.

(ii) They are both well described by lowest-order perturbation theory above the chiral phase transition for temperatures $T \gtrsim 1.75 T_{\mathrm{c}}$.

(iii) Their structure changes drastically below $T_{\mathrm{c}}$.

This paper is organized as follows. In sect. 2 we discuss some basic properties of the finite-temperature quark correlation functions in the euclidean time formalism. Sect. 3 deals with a perturbative calculation of the fermion self-energy at finite temperature on the lattice. Monte Carlo calculations of the quark correlation functions on euclidean lattices for four flavour QCD are presented in sect. 4. Finally we give our conclusions in sect. 5. An appendix is devoted to the details of the perturbative calculation of the fermion self-energy on the lattice.

\section{Temporal and spatial correlation functions}

Finite-temperature lattice simulations are usually performed on asymmetric lattices of size $N_{\tau} \times N_{\sigma}^{3}$. The shorter direction, $N_{\tau}$, is related to the temperature, $T$, through the lattice spacing, $a$, by the relation $T=1 / a N_{\tau}{ }^{\star}$. In $\mathrm{QCD}$, one can

* In the following we will set the lattice spacing to unity, $a \equiv 1$. 
construct hadronic correlation functions for separations in either the spatial or temporal directions. In lattice units we can write

$$
\begin{aligned}
& G_{\mathrm{s}}^{H}\left(x_{3}, \tilde{\boldsymbol{p}}\right)=\sum_{\tilde{\boldsymbol{x}}=\left(x_{0}, x_{1}, x_{2}\right)} \mathrm{e}^{i \tilde{\boldsymbol{p}} \cdot \overline{\boldsymbol{x}}}\left\langle H\left(\tilde{\boldsymbol{x}}, x_{3}\right) H^{\dagger}(\tilde{\mathbf{0}}, 0)\right\rangle, \\
& G_{\mathrm{t}}^{H}\left(x_{0}, \boldsymbol{p}\right)=\sum_{\boldsymbol{x}=\left(x_{1}, x_{2}, x_{3}\right)} \mathrm{e}^{i \boldsymbol{p} \cdot \boldsymbol{x}}\left\langle H\left(x_{0}, \boldsymbol{x}\right) H^{\dagger}(0, \boldsymbol{0})\right\rangle .
\end{aligned}
$$

Here $\tilde{\boldsymbol{p}}=\left(p_{0}, p_{1}, p_{2}\right), \boldsymbol{p}=\left(p_{1}, p_{2}, p_{3}\right)$, and $H$ denotes a hadronic operator of fixed quantum numbers. Note that for bosons $p_{0}=2 n \pi T$, whereas for fermions $p_{0}=(2 n+1) \pi T$ where $n=0, \pm 1, \pm 2, \ldots$ The expectation value of the product $H H^{\dagger}$, above, can be expressed in terms of quark propagators.

In the past, lattice studies have concentrated on analyses of spatial correlation functions *. This is due to technical reasons. Since $N_{\tau}$ is usually small, and (anti) periodicity of field configurations halves the possible number of independent measurements of a correlation function, the long-distance behaviour of correlators is difficult to measure in the temporal directions. This restriction is not so stringent for spatial correlation functions. On the other hand, we will show that the presently available spatial volumes, $N_{\sigma} \sim(2-3) N_{\tau}$, severely restrict the analysis of the low-momentum structure of these correlation functions.

The screening masses calculated from the exponential decay of $G_{\mathrm{s}}^{H}\left(x_{3}, \tilde{\mathbf{0}}\right)$ at large $x_{3}$ above the critical temperature are close to multiples of the Matsubara frequency $\pi T$; a result obtainable in a theory of non-interacting fermions. This large zeroth-order value makes it difficult to extract the corrections arising at higher orders of perturbation theory $[6,15]$. The situation is quite different for temporal correlation functions. Their exponential decay can, with perfect generality, be related to the singularities of the spectral function. Hence they can be easily related to the real-time correlator and are directly related to the spectrum of physical excitations $[9,10]$. In this section we discuss the structure of spatial and temporal quark correlation functions. These are the basic ingredients entering the correlators in eq. (1).

The quark correlators, analogous to the hadronic correlation functions of eq. (1) are related to the momentum-space propagator $S\left(p_{0}, p\right)$ through Fourier transformations:

$$
\begin{aligned}
& G_{\mathrm{t}}(\tau, \boldsymbol{p})=T \sum_{p_{0}} \mathrm{e}^{i p_{0} \tau} S\left(p_{0}, \boldsymbol{p}\right) \\
& G_{\mathrm{s}}(z, \tilde{\boldsymbol{p}})=\int \frac{\mathrm{d} p_{3}}{2 \pi} \mathrm{e}^{i p_{3} z} S\left(p_{0}, \boldsymbol{p}\right) .
\end{aligned}
$$

* Some result for temporal hadronic correlation functions on anisotropic lattices have, however, been presented in ref. [14]. 
The spectral representation of the finite-temperature propagator,

$$
S\left(p_{0}, \boldsymbol{p}\right)=i \int \frac{\mathrm{d} \omega}{2 \pi} \frac{\rho(\omega, \boldsymbol{p})}{i p_{0}-\omega},
$$

can be used to relate these two correlation functions to each other, and to the real-time correlator. In particular, the temporal propagator is obtained by combining eqs. (2) and (3). The summation over the momenta $p_{0}$ can be performed by the usual trick of contour integration, since the allowed values are obtained as the poles of the Fermi function $1 /(1+\exp (\omega / T))$. Thus the spectral representation of the temporal correlation function can be written as the contour integral

$$
G_{\mathrm{t}}(\tau, \boldsymbol{p})=\oint \frac{\mathrm{d} \omega}{2 \pi i} \rho(\omega, \boldsymbol{p}) \frac{\mathrm{e}^{\omega \tau}}{1+\mathrm{e}^{\omega / T}}
$$

where the contour avoids all the poles of the Fermi function. Hence $G_{t}(\tau, p)$ contains information only on the singularities of the spectral function, and thus directly on the quasi-particle spectrum of the theory. This is not true of the spatial correlator as can be demonstrated by writing the spectral representation of $G_{\mathrm{s}}$ which follows from eqs. (2) and (3). An appropriately chosen contour integral representation of eq. 3 must include only one pole of the Fermi function. For the lowest allowed momentum $\tilde{p}_{\min }=(\pi T, 0,0)$, the Matsubara frequency shows up in the long-distance behaviour of this correlator. Thus, genuine spectral information is harder to extract from this correlator.

The example of a free-field theory clarifies these statements. One can write the spectral density in the form

$$
\rho_{\mathrm{ff}}(\omega, p)=\frac{-i \pi}{2 \omega}\left(i \gamma_{0} \omega+i \gamma \cdot p+m\right)\left(\delta\left(\omega-\omega_{p}\right)+\delta\left(\omega+\omega_{p}\right)\right)
$$

for fermions of mass $m$, where $p=|p|$ and $\omega_{p}=\sqrt{p^{2}+m^{2}}$. Using eq. (4), one finds

$$
\begin{aligned}
G_{\mathrm{t}}(\tau, \boldsymbol{p})= & \frac{i}{2 \cosh \left(\omega_{p} / 2 T\right)} \\
& \times\left[i \gamma_{0} \cosh \left(\omega_{p}\left(\tau-\frac{1}{2} T\right)\right)+\left(\frac{i \gamma \cdot \boldsymbol{p}+m}{\omega_{p}}\right) \sinh \left(\omega_{p}\left(\tau-\frac{1}{2} T\right)\right)\right] .
\end{aligned}
$$

In contrast, the spatial correlator decays with an effective mass $\Omega=\sqrt{m^{2}+\pi^{2} T^{2}}$, and takes the form

$$
G_{\mathrm{s}}\left(z, \tilde{\boldsymbol{p}}_{\min }\right)=\mathrm{e}^{-|z| \Omega}\left[\gamma_{0}(i \pi T)+\gamma_{3}(\Omega / 2)+m\right] / \Omega .
$$

It is a trivial job to transcribe these results to a lattice. 
We turn now to the corresponding QCD problem. The temporal quark propagator in high-temperature perturbation theory has been extensively analysed during the last years [11-13]. The fermion self-energy has been computed to $O\left(g^{2}\right)$ [16]. In the high-temperature limit, the leading contribution is gauge invariant and is shown to be of $\mathrm{O}\left(g^{2} T^{2}\right)$. For vanishing bare quark mass, the renormalized propagator can be written as

$$
S^{-1}\left(p_{0}, \boldsymbol{p}\right)=\gamma_{0} D_{0}\left(p_{0}, \boldsymbol{p}\right)+i \gamma \hat{p} D_{\mathrm{s}}\left(p_{0}, \boldsymbol{p}\right)
$$

where $\hat{p}=\boldsymbol{p} /|\boldsymbol{p}|$. The functions $D_{0, \mathrm{~s}}$ are in general quite involved [11], but take on the following simple form when only the leading corrections are retained:

$$
\begin{aligned}
& D_{0}\left(p_{0}, \boldsymbol{p}\right)=i p_{0}-\frac{m_{\mathrm{eff}}^{2}}{2 p} \ln \left(\frac{i \underline{p}_{0}+p}{i p_{0}-p}\right) \\
& D_{\mathrm{s}}\left(p_{0}, \boldsymbol{p}\right)=p+\frac{m_{\mathrm{eff}}^{2}}{p}\left(1-\frac{i p_{0}}{2 p} \ln \left(\frac{i p_{0}+p}{i p_{0}-p}\right)\right)
\end{aligned}
$$

The effective quark mass is given by

$$
m_{\mathrm{eff}}^{2}=\frac{1}{8}\left(\frac{N^{2}-1}{2 N}\right) g^{2} T^{2} .
$$

Analysis of the real-time theory gives similar results.

For non-zero momenta $\boldsymbol{p}$, the spectral density at finite temperature has two poles $[16], \omega_{+}(p)$ and $\omega_{-}(p)$. These coincide at vanishing three-momentum, $\omega_{+}(\mathbf{0})=\omega_{-}(\mathbf{0})=m_{\text {eff }}$. In fact, for $p=0$, one finds

$$
\begin{aligned}
& D_{0}\left(p_{0}, 0\right)=i p_{0}\left(1+\frac{m_{\text {eff }}^{2}}{p_{0}^{2}}\right) \\
& D_{\mathrm{s}}\left(p_{0}, 0\right)=0 .
\end{aligned}
$$

After analytic continuation, $D_{0}$ correctly gives rise to a pole $p_{0}=m_{\mathrm{eff}}$. This is reflected in the zero-momentum temporal correlation function. For non-vanishing three-momentum the latter is given by the expression [11]

$$
\begin{aligned}
G_{\mathrm{t}}(\tau, \boldsymbol{p})= & \int_{0}^{\infty} \mathrm{d} \omega \rho_{0}(\omega, \boldsymbol{p}) \gamma_{0} \cosh \left(\omega\left(\tau-\frac{1}{2} T\right)\right) \\
& +\rho_{\mathrm{s}}(\omega, \boldsymbol{p}) \gamma \hat{p} \sinh \left(\omega\left(\tau-\frac{1}{2} T\right)\right)
\end{aligned}
$$


where the components, $\rho_{0, \mathrm{~s}}$, of the spectral density receive contributions from the above-mentioned poles, and a cut for $0 \leqslant \omega \leqslant p$,

$$
\rho_{0, \mathrm{~s}}=A\left[\delta\left(\omega-\omega_{+}\right)+\delta\left(\omega-\omega_{-}\right)\right]+B \Theta(p-\omega) .
$$

The cut arises from the logarithms in eq. (9). The residues $A$ and $B$ are discussed in ref. [11]. It is easy to see that in the limit, $g^{2}=0$, these reduce to the free-field spectral density in eq. (5).

The spatial correlation function behaves in a different manner. Since $\tilde{\boldsymbol{p}}_{\min }$ is non-zero, the pole contribution now includes a part due to the Matsubara frequency, just as in the free theory. Moreover, $D_{\mathrm{s}}$ is no longer identically zero, and the cut visible there, eq. (9), also influences the behaviour of $G_{\mathrm{s}}$. For vanishing spatial momenta $\left(p_{1}=p_{2}=0\right)$ one finds

$$
\begin{aligned}
G_{\mathrm{s}}\left(z, p_{0}, 0,0\right) & =\gamma_{0} \int_{p_{0}}^{\infty} \mathrm{d} \omega \tilde{\rho}(\omega) \mathrm{e}^{-\omega z}, \\
\tilde{\rho}(\omega) & =\frac{1}{\pi} \operatorname{Im}\left\{D_{\mathrm{s}}(p) /\left[D_{0}^{2}(p)+D_{\mathrm{s}}^{2}(p)\right]\right\},
\end{aligned}
$$

where we have used the abbreviation $p=\left(p_{0}, 0,0, i \omega\right)$.

In conclusion, we note that the behaviour of the spatial and temporal correlation functions is quite different even in the non-interacting theory. When the effect of interactions is taken into account, further differences between the two can be observed. Perturbative corrections lead to small modifications of the propagators in the high-temperature limit. These modifications remain small for the spatial correlation function, because of the cutoff imposed by the Matsubara frequency. In the temporal correlator, on the other hand, these can become important when the screening mass, $m_{\text {eff }}$, gets large enough.

\section{The fermion propagator on the lattice}

Let us now consider the fermion propagator on the lattice. For simplicity, we discuss here the case of naive fermions. The lattice propagator is given by

$$
S_{\mathrm{L}}^{-1}(p)=S_{\mathrm{L}, 0}^{-1}(p)+\Sigma_{\mathrm{L}}(p)
$$

with $p=\left(p_{0}, p\right)$ and

$$
S_{\mathrm{L}, 0}^{-1}(p)=i \sum_{k=0}^{3} \gamma_{k} \sin \left(p_{k}\right)+m
$$



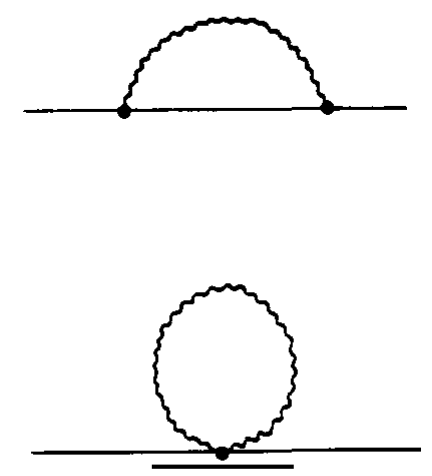

Fig. 1. Feynman diagrams contributing to the fermion self-energy at $O\left(g^{2}\right)$ on the lattice. Only the first one exists in the continuum formulation.

denoting the free propagator for quarks with bare mass $m$. The lattice self-energy correction may be written as

$$
\Sigma_{\mathrm{L}}(p)=g^{2} C_{\mathrm{F}}\left[i \sum_{k=0}^{3} \gamma_{k} \tilde{\Sigma}_{k}(p)+m \tilde{\Sigma}_{m}(p)\right]
$$

with $C_{\mathrm{F}}=\left(N^{2}-1\right) / 2 N$. It receives contributions from the two diagrams shown in fig. 1. The complete $\mathrm{O}\left(g^{2}\right)$ result in discussed in the appendix. Here we will consider only that part of the self-energy correction, which gives rise to the leading $\mathrm{O}\left((g T)^{2}\right)$ high-temperature contribution in the continuum limit, eq. (9). This comes from the second term in (A.10). The lattice computation yields

$$
\begin{aligned}
& D_{0}^{(\mathrm{L})}\left(p_{0}, \boldsymbol{p}\right)=\sin \left(p_{0}\right)\left(1+g^{2} C_{\mathrm{F}} \int_{q} \frac{\left(n_{\mathrm{F}}+n_{\mathrm{B}}\right) f_{0}\left(x_{+}\right)}{8 \omega_{\mathrm{B}} \sqrt{\omega_{\mathrm{B}}^{2}+1} \sqrt{\omega_{\mathrm{F}}^{2}+1}}\right) \\
& D_{k}^{(\mathrm{L})}\left(p_{0}, \boldsymbol{p}\right)=\sin \left(p_{k}\right)+g^{2} C_{\mathrm{F}} \int_{q} \frac{\left(n_{\mathrm{F}}+n_{\mathrm{B}}\right) f_{k}\left(x_{+}\right) \sin \left(q_{k}\right)}{8 \omega_{\mathrm{F}} \omega_{\mathrm{B}} \sqrt{\omega_{\mathrm{B}}^{2}+1} \sqrt{\omega_{\mathrm{F}}^{2}+1}}, \\
& D_{m}^{(\mathrm{L})}\left(p_{0}, \boldsymbol{p}\right)=1+g^{2} C_{\mathrm{F}} \int_{q} \frac{\left(n_{\mathrm{F}}+n_{\mathrm{B}}\right) f_{m}\left(x_{+}\right)}{8 \omega_{\mathrm{F}} \omega_{\mathrm{B}} \sqrt{\omega_{\mathrm{B}}^{2}+1} \sqrt{\omega_{\mathrm{F}}^{2}+1}} .
\end{aligned}
$$

The integrals appearing in these expressions define the coefficients $\tilde{\Sigma}$ of the self-energy correction, eq. (17). Detailed definitions of the functions involved are given in the appendix. We note here that the lattice analogue to the $m_{\text {eff }}^{2} / p_{0}^{2}$ term in the continuum expression for $D_{0}$, eq. (9), is a complicated integral, which has a rather complex dependence on $p_{0}$, even for vanishing momentum $\boldsymbol{p}$. A unique definition of $m_{\text {eff }}$ on the lattice is thus possible only in the continuum limit. 
Including only these " $\mathrm{O}((g T))$ " lattice corrections in the renormalized propagator yields the temporal and spatial quark correlation function:

$$
\begin{aligned}
& G_{\mathrm{t}}\left(x_{0}, \boldsymbol{p}\right)=\frac{-i}{N_{\tau}} \sum_{i_{0}=0}^{N_{\tau}-1} \frac{\mathrm{e}^{i p_{0} x_{0}}}{\gamma_{0} D_{0}^{(\mathrm{L})}+\sum_{k} \gamma_{k} D^{(\mathrm{L})}+m D_{m}^{(\mathrm{L})}}, \\
& G_{\mathrm{s}}\left(x_{3}, \tilde{\boldsymbol{p}}\right)=\frac{-i}{N_{\sigma}} \sum_{i_{3}=0}^{N_{\sigma}-1} \frac{\mathrm{e}^{i p_{3} x_{2}}}{\gamma_{0} D_{0}^{(\mathrm{L})}+\sum_{k} \gamma_{k} D_{k}^{(\mathrm{L})}+m D_{m}^{(\mathrm{L})}},
\end{aligned}
$$

In the $g^{2} \rightarrow 0$ limit the correlation functions $G_{\mathrm{t}}$ and $G_{\mathrm{s}}$ are just the free fermion correlation functions. For staggered as well as naive lattice fermions their real parts are given by

$$
G_{\mathrm{t}}\left(x_{0}, \boldsymbol{p}\right)=\left\{\begin{array}{l}
\cosh \left(E_{\mathrm{t}}\left(x_{0}-N_{\tau} / 2\right)\right)\left[\cosh \left(E_{\mathrm{t}} N_{\tau} / 2\right) \cosh \left(E_{\mathrm{t}}\right)\right]^{-1} \\
\quad\left(x_{0} \text { odd }\right) \\
-2 m \sinh \left(E_{\mathrm{t}}\left(x_{0}-N_{\tau} / 2\right)\right)\left[\cosh \left(E_{\mathrm{t}} N_{\tau} / 2\right) \sinh \left(2 E_{\mathrm{t}}\right)\right]^{-1} \\
\quad\left(x_{0} \text { even }\right)
\end{array}\right.
$$

and

$$
G_{\mathrm{s}}\left(x_{3}, \tilde{\boldsymbol{p}}\right)=\left\{\begin{array}{l}
\sinh \left(E_{\mathrm{s}}\left(x_{3}-N_{\sigma} / 2\right)\right)\left[\sinh \left(E_{\mathrm{s}} N_{\sigma} / 2\right) \cosh \left(E_{\mathrm{s}}\right)\right]^{-1} \\
\quad\left(x_{3} \text { odd }\right) \\
2 m \cosh \left(E_{\mathrm{s}}\left(x_{3}-N_{\sigma} / 2\right)\right)\left[\sinh \left(E_{\mathrm{s}} N_{\sigma} / 2\right) \sinh \left(2 E_{\mathrm{s}}\right)\right]^{-1} \\
\left(x_{3} \text { even }\right)
\end{array}\right.
$$

with

$$
\begin{aligned}
& E_{\mathrm{t}, \mathrm{s}}=\ln \left(\omega_{\mathrm{t}, \mathrm{s}}+\sqrt{\omega_{\mathrm{t}, \mathrm{s}}^{2}+1}\right) \\
& \omega_{\mathrm{t}}^{2}=\sum_{k=1}^{3} \sin ^{2}\left(p_{k}\right)+m^{2}, \quad \omega_{\mathrm{s}}^{2}=\sum_{k=0}^{2} \sin ^{2}\left(p_{k}\right)+m^{2}
\end{aligned}
$$

We note that the lattice correlation functions oscillate between even and odd sites, vanishing on even sites for vanishing bare quark mass as long as $N_{\tau}$ stays finite. Moreover, in this limit, for vanishing spatial momenta, the temporal correlation function is constant, while the lowest-momentum spatial correlation function decays exponentially with a screening mass determined by the lowest Matsubara frequency. On a finite temporal lattice this becomes

$$
E_{\mathrm{s}, \min }=\ln \left(\sqrt{\sin ^{2}\left(\pi / N_{\tau}\right)+m^{2}}+\sqrt{\sin ^{2}\left(\pi / N_{\tau}\right)+m^{2}+1}\right) .
$$


In order to get some feeling for the finite-size effects to be expected in lattice calculations, we determine the effective quark mass and spatial screening mass from the lattice propagators for the case of vanishing bare quark mass. The effective quark mass can be obtained from $\tilde{\Sigma}_{0}\left(p_{0}, 0\right)$. As in the continuum formulation, one can deduce from eq. (18) that the self-energy for vanishing three-momentum develops a $1 / \sin \left(p_{0}\right)$ singularity in the infinite-volume limit. For finite temporal extents, however, the dependence of $D_{0}^{(\mathrm{L})}\left(p_{0}, \mathbf{0}\right)$ on $\sin \left(p_{0}\right)$ is still quite complicated and one thus cannot simply factor out a $p_{0}$-independent term, which could be uniquely identified as the lattice analogue of the effective quark mass. We thus discuss two definitions of $m_{\text {eff }}$ on the lattice, which are related to the structure of the propagator and correlation functions, respectively. We may use the $\mathrm{O}\left((g T)^{2}\right)$ contribution to the self-energy, eq. (18), calculated for the smallest possible $p_{0}\left(=\pi / N_{\tau}\right)$ to define an approximation to $m_{\text {eff }}$ on finite lattices.

$$
m_{\mathrm{eff}, \mathrm{L}}^{2} / T^{2}=g^{2} C_{\mathrm{F}} c\left(N_{\tau}, N_{\sigma}\right), \quad c\left(N_{\tau}, N_{\sigma}\right)=\sin \left(\pi / N_{\tau}\right) \tilde{\Sigma}_{0}\left(\pi / N_{\tau}, 0\right)
$$

Alternatively one can define an effective quark mass through the exponential decay of the temporal correlation function calculated to $\mathrm{O}\left((g T)^{2}\right)$ as given in eqs. (18) and (19). Using a simple cosh-fit to $G_{\mathfrak{t}}\left(x_{0}, \mathbf{0}\right)$ we can extract a lattice size dependent mass, which we write in the form given by eq. (24) to define the coefficient $c\left(N_{\tau}, N_{\sigma}\right)$. We note that this procedure corresponds to the approach one usually follows in the analysis of the propagator obtained from lattice Monte Carlo simulations.

Results for the coefficient $c\left(N_{\tau}, N_{\sigma}\right)$ obtained through the above described methods are summarized in tables 1 and 2 . We note that on spatially infinite lattices $c\left(N_{\tau}, \infty\right)$ rapidly approaches the continuum result, $c(\infty, \infty)=1 / 8$ (table 1 ). Indeed, already for $N_{\tau} \geq 8$ the deviations are small. The finite-size effects are, however, much larger for finite $N_{\sigma}$. This becomes clear from table 2 , which shows

TABle 1

The coefficient $c\left(N_{\tau}, N_{\sigma}\right)$ of the approximations for the effective quark mass on spatially infinite lattices

\begin{tabular}{rcc}
$N_{\tau}$ & $\begin{array}{c}c\left(N_{\tau}, \infty\right) \\
\text { from eq. (24)] }\end{array}$ & $\begin{array}{c}c\left(N_{\tau}, \infty\right) \\
\text { [from eq. (19)] }\end{array}$ \\
\hline 4 & 0.1844 & - \\
8 & 0.1356 & 0.1211 \\
36 & 0.1271 & 0.1245 \\
$\infty$ & 0.1254 & 0.1249 \\
& 0.1250 & 0.1250 \\
\hline
\end{tabular}

The second column shows results obtained from the definition given in eq. (24). The third column is based on a cosh-fit to the temporal correlation function, eq. (19), which are shown in fig. 2 for $N_{\tau}=8$. The corresponding result from $\mathrm{O}\left((g T)^{2}\right)$ perturbation theory in the continuum is $c(\infty, \infty)=1 / 8$. 
TABLE 2

The coefficients $x\left(N_{\tau}, N_{\sigma}\right)$ for various values of $N_{\sigma}$ and $N_{\tau}=8$ and 16 . We note that $c\left(N_{\tau}, N_{\sigma}\right)$ approximately scales with $N_{\sigma} / N_{\tau}$

\begin{tabular}{rcccc}
\hline$N_{\sigma}$ & $\begin{array}{c}c\left(8, N_{\sigma}\right) \\
\text { [from eq. (24)] }\end{array}$ & $\begin{array}{c}c\left(16, N_{\sigma}\right) \\
\text { [from eq. (24)] }\end{array}$ & $\begin{array}{c}c\left(8, N_{\sigma}\right) \\
\text { [from eq. (19)] }\end{array}$ & $\begin{array}{c}c\left(16, N_{\sigma}\right) \\
\text { [from eq. (19)] }\end{array}$ \\
\hline 16 & 0.03770 & - & 0.03201 & - \\
32 & 0.07814 & 0.03276 & 0.06985 & 0.03186 \\
64 & 0.10577 & 0.07233 & 0.09551 & 0.07071 \\
128 & 0.12059 & 0.09872 & 0.10926 & 0.09659 \\
$\infty$ & 0.1356 & 0.1271 & 0.1211 & 0.1245 \\
\hline
\end{tabular}
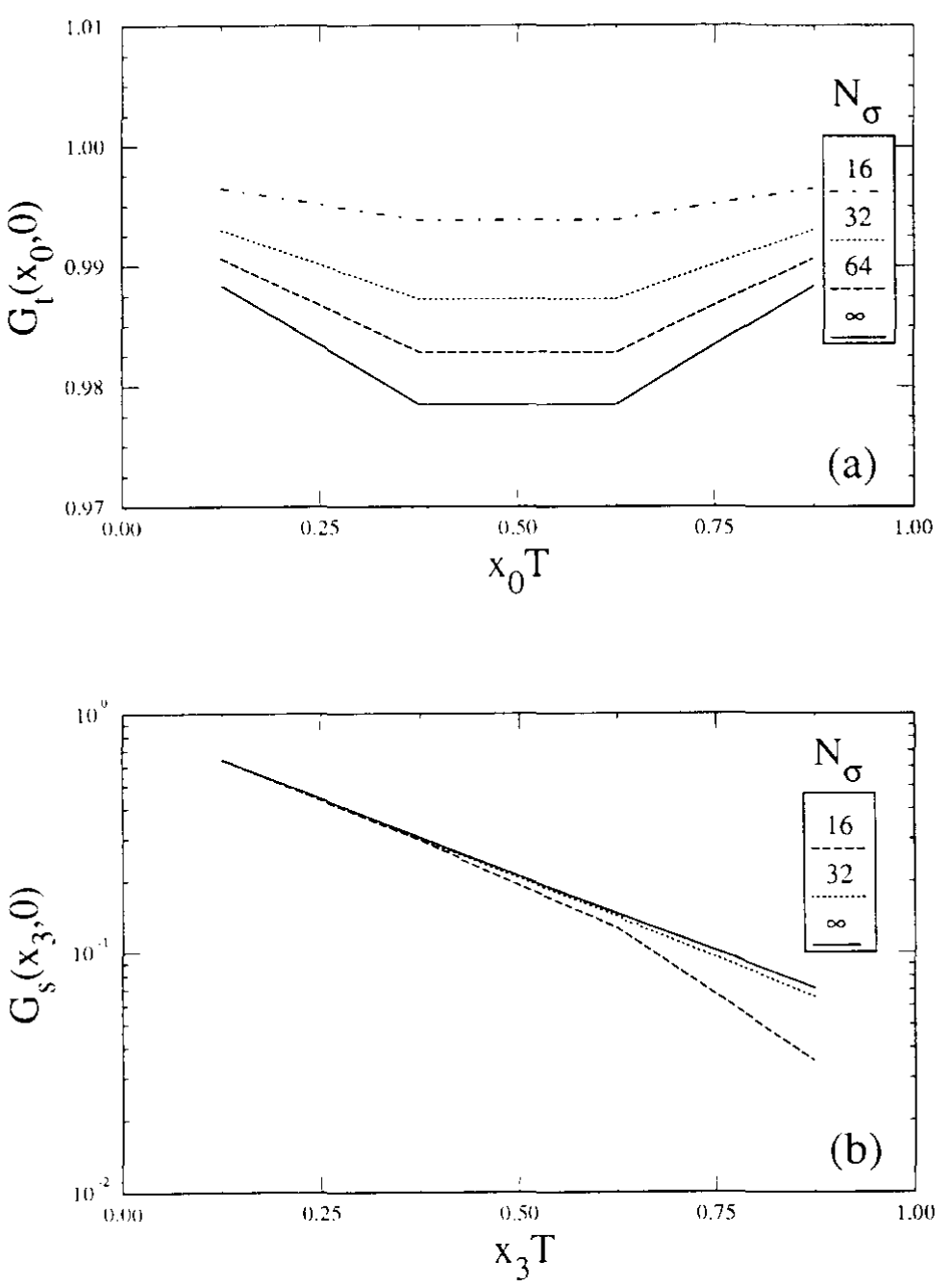

Fig. 2. Temporal (a) and spatial (b) quark correlation functions calculated to $\mathrm{O}\left((g T)^{2}\right)$ for $g^{2}=1$ and $m=0$ on finite lattices of size $N_{\tau} \times N_{\sigma}^{3}$ with $N_{\tau}=8$ and various values of $N_{\sigma}$. 
that $N_{\sigma} / N_{\tau}$ must be large in order for us to obtain good estimates of $m_{\text {eff }}$ on finite lattices.

In fig. 2 we compare the lattice correlation functions for various values of $N_{\sigma}$ and fixed $N_{\tau}=8$ with the corresponding infinite-volume result. This too shows that rather large spatial lattices are needed to approximate the infinite-lattice result for temporal correlation functions. Note, however, the scale in fig. 2a. The deviations from the free-field behaviour, $G_{\mathrm{t}}\left(x_{0}, \mathbf{0}\right)=1$, due to $\mathrm{O}\left((g T)^{2}\right)$ corrections are only on the $1 \%$ level even for $g^{2} \simeq 1$. Consequently these hardly show up in fig. $2 b$ for the spatial correlation functions which are entirely dominated by the large screening mass of the free propagator and only for $x_{3} \sim N_{\sigma} / 2$ do deviations due to the lattice periodicity show up.

\section{Monte Carlo data}

We have studied quark correlation functions at finite temperature on the lattice for four-flavour QCD. The Monte Carlo data have been obtained from an analysis of gauge field configurations generated by the $\mathrm{MT}_{\mathrm{c}}$-collaboration in order to determine the critical temperature in four-flavour QCD with light quarks of bare mass $m=0.01$ on an $8 \times 16^{3}$ lattice [17]. Details of these and the analysis of hadronic correlation functions can be found in refs. [6,17]. We have added a simulation at $\beta=6.5$ to this data sample. For orientation we note that the chiral phase transition on lattices of this size was found to occur at $\beta_{c}=5.15 \pm 0.05$. This was signalled by two Monte Carlo runs with long-lived metastable states at this coupling. Earlier calculations for four-flavour QCD on lattices with $N_{\tau}=4$ [18] as well as spectrum calculations at $\beta=5.35$ [19] indicate that a change of the coupling by $\Delta \beta \simeq 0.2$ corresponds roughly to a change in the temperature by a factor of two.

The quark propagator is a gauge-variant quantity although its pole is not. We thus have to fix the gauge in order to analyze the quark correlation functions. We have used a Landau gauge fixing. This was implemented by first fixing a complete axial gauge on the configurations $\left\{U_{i, \mu}\right\}$ and then minimizing the functional

$$
H_{\mathrm{L}}=\sum_{i, \mu}\left(1-\frac{1}{N} \operatorname{Re} \operatorname{Tr} G_{i} U_{i, \mu} G_{i+\mu}\right)
$$

under variation of the $V$ gauge group elements $\left\{G_{i}\right\}$ living on sites of the lattice. Although the general minimisation problem does not have a unique solution, when the distribution of link variables is strongly peaked, there are very few minima. In this case a unique solution may be obtained by first fixing to the axial gauge. Nonetheless, the existence of Gribov copies which can contribute to the quark propagator [20] may show up in future high-statistics simulations. 
After gauge fixing we have studied the temporal and spatial correlation functions,

$$
\begin{aligned}
& G_{t}\left(x_{0}, \boldsymbol{p}\right)=\sum_{\boldsymbol{x}=\left(x_{1}, x_{2}, x_{3}\right)} \mathrm{e}^{i \boldsymbol{x} \cdot \boldsymbol{p}}\left\langle\bar{\chi}_{0} \chi_{\left(x_{0}, \boldsymbol{x}\right)}\right\rangle, \\
& G_{\mathrm{s}}\left(x_{3}, \tilde{\boldsymbol{p}}\right)=\sum_{\tilde{\boldsymbol{x}}=\left(x_{0}, x_{1}, x_{2}\right)} \mathrm{e}^{i \tilde{x} \cdot \tilde{\boldsymbol{p}}}\left\langle\bar{\chi}_{0} \chi_{\left(\overline{\boldsymbol{x}}, x_{3}\right)}\right\rangle,
\end{aligned}
$$

where $\left\langle\bar{\chi}_{x} \chi_{y}\right\rangle$ denotes the quark propagator for staggered fermions between lattice sites $x$ and $y$. For each value of $\beta$ we have typically analyzed 50 configurations, using 4 source points on each (at $\beta=6.5$ we had only 15 configurations). Errors have been estimated by dividing the total data sample into four blocks (three blocks at $\beta=6.5$ ) and taking the variance of the results obtained on these blocks.

As discussed in the previous sections, we expect that the zero-momentum correlation functions in the high-temperature phase drop exponentially with a mass given by the lowest Matsubara frequency or the effective quark mass in the spatial and temporal directions, respectively. In fig. 3 we show both correlation functions at $\beta=5.3$, i.e. in the plasma phase at a temperature which is approximately $1.75 T_{\mathrm{c}}$. The different behaviour of the temporal and spatial correlators is clearly seen. We also note the strong oscillatory behaviour between even and odd sites, which is characteristic for the free fermion propagator on lattices with small spatial and temporal extent (eqs. (21) and (20)). The different functional form of the propagator on even and odd sites ( $\cosh / \sinh )$, reflecting the (anti-)periodicity of the lattice, influences the structure of the correlation functions even at short distances (when $N_{\sigma}$ is not much larger than $N_{\tau}$ ). The free propagators on finite lattices are also shown in fig. 3. We note that the free correlation functions on even sites are proportional to the bare quark mass. For this reason they are more sensitive to changes in the effective quark mass as is obvious from fig. 3 .

Clearly the quark propagators at temperatures as low as $1.75 T_{\mathrm{c}}(\beta=5.3)$ are already quite close to the free propagators. This changes drastically below $T_{\mathrm{c}}$ as can be seen in fig. 4 , where we show the correlation functions at $\beta=5.1$, i.e. in the chiral symmetry broken phase. The oscillations between even and odd sites decrease, which is due to a larger effective screening mass. In particular the temporal propagator now shows a strong curvature. Although it is not apparent from the figure (due to the different lattice size in spatial and temporal direction), the screening masses extracted from the slopes of the spatial and temporal propagators agree, within errors (see table 3 ) below $T_{\mathrm{c}}$. Moreover, we note already here that these screening masses are compatible with half the $\rho$-mass calculated at zero temperature $[19,21]$.

We have determined screening masses from the exponential decay of the spatial and temporal correlation functions using an ansatz for the fit which is motivated by 
the form of the free propagators, i.e. we use three-parameter fits to the complete correlation functions *

$$
\begin{aligned}
G_{\mathrm{t}}\left(x_{0}, \boldsymbol{p}\right)= & \frac{a_{\mathrm{t}}}{2}\left(1-(-1)^{x_{0}}\right) \cosh \left(m_{\mathrm{t}}\left(x_{0}-N_{\tau} / 2\right)\right) \\
& +\frac{b_{\mathrm{t}}}{2}\left(1+(-1)^{x_{0}}\right) \sinh \left(m_{\mathrm{t}}\left(x_{0}-N_{\tau} / 2\right)\right), \\
G_{\mathrm{s}}\left(x_{3}, \tilde{\boldsymbol{p}}\right)= & \frac{a_{\mathrm{s}}}{2}\left(1-(-1)^{x_{3}}\right) \sinh \left(m_{\mathrm{s}}\left(x_{3}-N_{\sigma} / 2\right)\right) \\
& +\frac{b_{\mathrm{s}}}{2}\left(1+(-1)^{x_{3}}\right) \cosh \left(m_{\mathrm{s}}\left(x_{3}-N_{\sigma} / 2\right)\right) .
\end{aligned}
$$

The screening masses and amplitudes obtained from fits to the zero-momentum correlation functions are summarized in table 3 . The screening masses $m_{\mathrm{t}}, m_{\mathrm{s}}$ are shown in fig. 5 . We note that, below $T_{c}$, the two are identical within statistical errors. At $T_{\mathrm{c}}$ we find in the chiral symmetry broken phase $m_{\mathrm{t}} \simeq m_{\mathrm{s}} \simeq 0.44$. This should be compared with the $\rho$-mass, which has been calculated at the same coupling ( $\beta=5.15)$ and for the same value of the bare quark mass $(m=0.01)$ on a large zero-temperature lattice [21]. This gave $m_{\rho}=0.93(5)$. The effective quark mass calculated in the chiral symmetry broken phase may thus be interpreted as a constituent quark mass, $m_{\mathrm{s}, \mathrm{t}} \simeq m_{\rho} / 2$.

Above $T_{\mathrm{c}}$ the screening masses are quite different. While $m_{\mathrm{s}} / T$ is close to $\pi$ and changes little across $T_{\mathrm{c}}, m_{\mathrm{t}}$ drops by a factor of nearly two at $T_{\mathrm{c}}$ and rapidly approaches small values. These are compatible with expectations from the $\mathrm{O}\left(g^{2}\right)$ perturbative calculations: $m_{\mathrm{t}} / T \sim \sqrt{g^{2} / 6}$, with $g^{2} \sim \mathrm{O}(1)$. We note, however, that the small variation of $m_{\mathrm{s}} / T$ at $T_{\mathrm{c}}$ seems to be accidental. It is due to the fact that for four-flavour QCD $m_{\rho} / 2$ turns out to be roughly equal to $\pi T_{\mathrm{c}}$. This is different, for instance, in quenched QCD, as the transition temperature is larger by factor 2 in that case. Similar effects have been noticed in the analysis of quenched and unquenched spatial meson correlation functions [6].

The values obtained for $m_{\mathrm{t}} / T$ in the chiral symmetric phase close to $T_{\mathrm{c}}$ ( $\beta=5.15$ (S) and 5.2) are still considerably larger than the perturbative results, i.e. $g(T) \simeq 4$ would be needed to describe the Monte Carlo data in terms of the perturbative formula for temperatures $T_{\mathrm{c}} \leqslant T \leqslant 1.5 T_{\mathrm{c}}$. This is also confirmed by an analysis of the ratio of even/odd amplitudes of the quark propagators, $b_{\mathrm{s}, \mathrm{t}} / a_{\mathrm{s}, \mathrm{t}}$, which are shown in fig. 6 for various values of $\beta$. Both ratios drop at $T_{\mathrm{c}}$ and start approaching the corresponding free field values, $b_{\mathrm{s}} / a_{\mathrm{s}} \sim 0.026$, for $N_{\tau}=8$ and $m=0.01$, and $b_{\mathrm{t}} / a_{\mathrm{t}}=1$, for $\beta \geqslant 5.3$.

* At $\beta=6.5$ we could only perform a two-parameter fit for the temporal correlation function. In that case we demanded the amplitudes to be equal, $a_{t}=b_{1}$. 

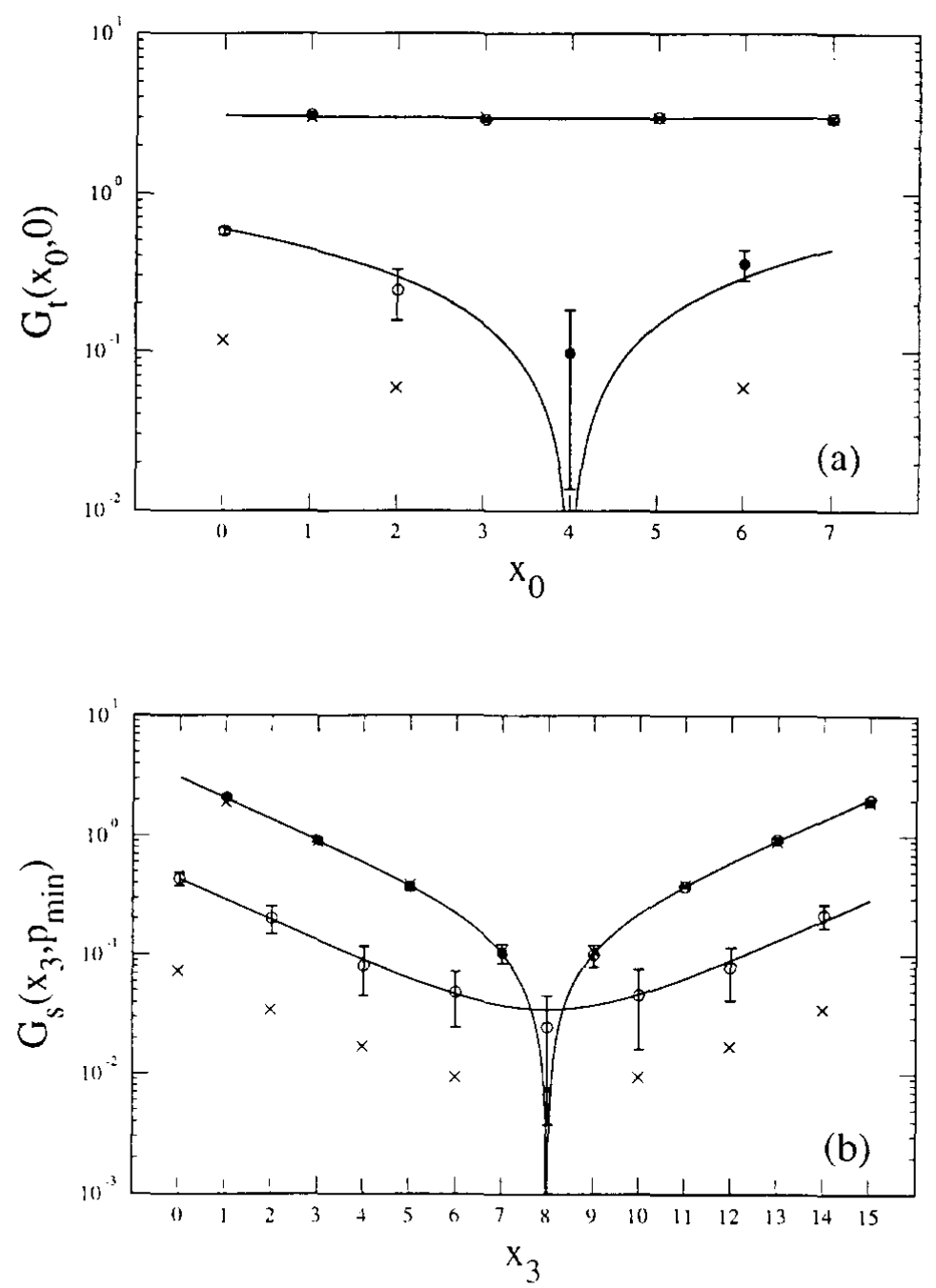

Fig. 3. The quark correlation function at $\beta=5.3$ in temporal (a) and spatial (b) direction of the $8 \times 16^{3}$ lattice. Here $p_{\min }=(\pi / 8,0,0)$ denotes the smallest possible momentum in the spatial correlation function. The two solid lines correspond to the even and odd site contributions to the fit function given in eq. (27). Also shown are the free quark correlation functions (crosses) calculated on a lattice of same size. These are on top of the measured data points on odd lattice sites. Filled circles correspond to negative values.

We have also studied the correlation functions at non-vanishing momentum, i.e. $\tilde{\boldsymbol{p}}=\left(\pi / N_{\tau}, p_{1}, 0\right)$ and $\boldsymbol{p}=\left(p_{1}, 0,0\right)$. Results for the dispersion relations $E_{\mathrm{t}, \mathrm{s}}\left(p_{1}\right)$ defined in eq. (22) are shown in fig. 7. Due to the periodicity of the lattice in spatial direction, and the doubling of fermion species, these dispersion relations are symmetric around $p_{1}=\pi / 2$. On a lattice of spatial extent $N_{\sigma}$ there are thus only $N_{\sigma} / 4$ independent momenta. In fig. 7 we show results for the momenta $p_{1}=2 \pi n / N_{\sigma}$ with $n=0,1, \ldots, N_{\sigma} / 4$. A comparison with the free fermion dispersion relation on this size lattice,

$$
E_{\mathrm{t}, \mathrm{s}}\left(p_{1}\right)=\operatorname{arcsinh}\left(\sqrt{m_{\mathrm{t}, \mathrm{s}}^{2}+\sin ^{2}\left(p_{1}\right)}\right)
$$



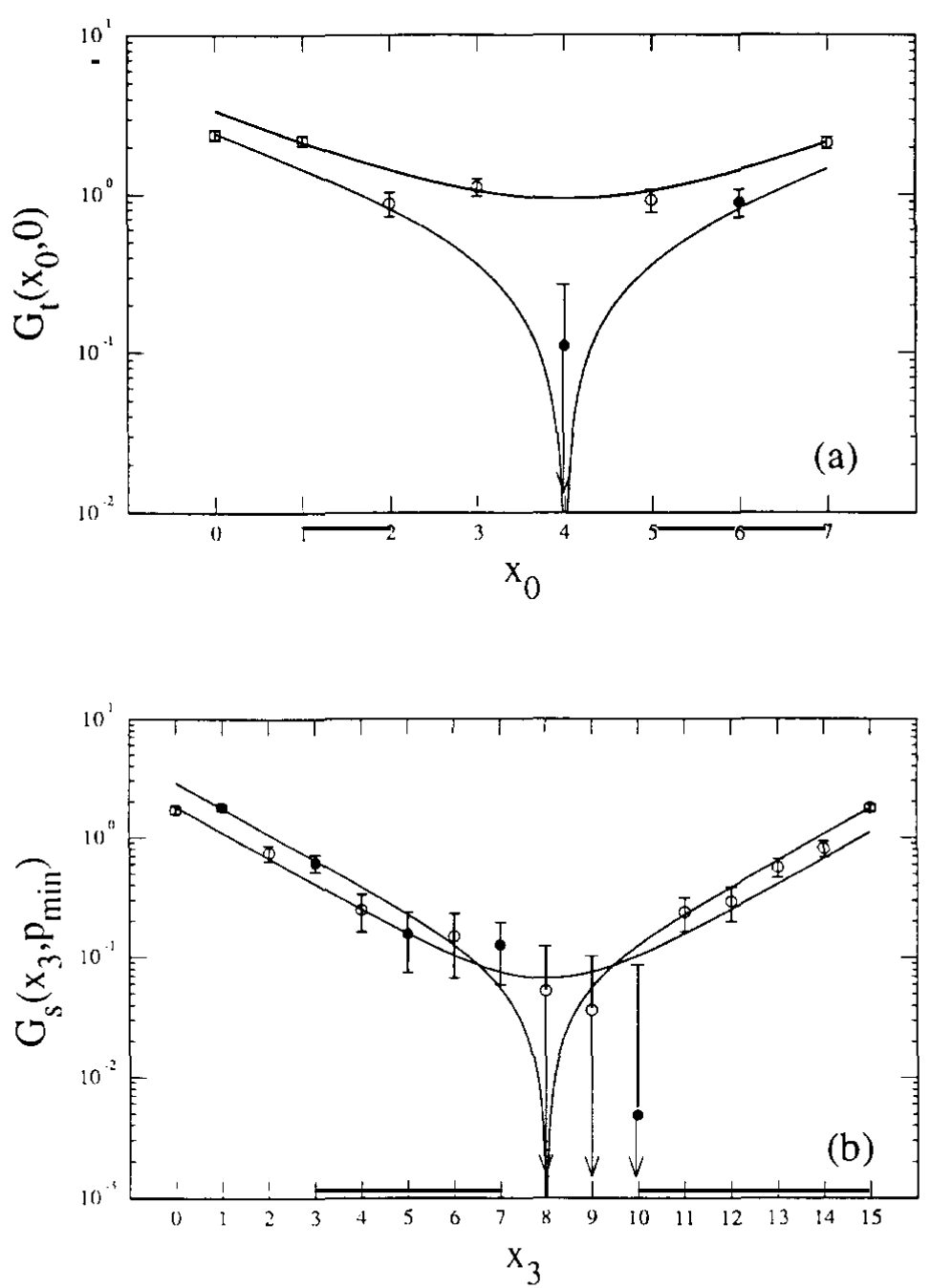

Fig. 4. Same as fig. 3 but for the quark correlation function at $\beta=5.1$.

with $m_{\mathrm{s}}=\sqrt{\sin ^{2}\left(\pi / N_{\tau}\right)+m^{2}}$ and $m_{\mathrm{t}} \equiv m=0.01$ shows that the spatial correlation functions agree quite well with this expression for all couplings. This is also true for the temporal dispersion relation at large momentum, where the influence of

TABLE 3

Screening masses extracted from temporal $\left(m_{\mathrm{t}}\right)$ and spatial $\left(m_{\mathrm{s}}\right)$ quark correlation functions as well as the amplitudes of the odd $\left(a_{\mathrm{s}, \mathrm{t}}\right)$ and even $\left(b_{\mathrm{s}, \mathrm{t}}\right)$ site fitting functions as defined in eq. (27). For $\beta=6.5$ we only have performed a two-parameter fit for the temporal correlation function, demanding $a_{\mathrm{t}}=b_{\mathrm{t}}$

\begin{tabular}{lllllll}
\hline \multicolumn{1}{c}{$\beta$} & \multicolumn{1}{c}{$m_{\mathrm{t}}$} & $m_{\mathrm{s}}$ & \multicolumn{1}{c}{$a_{\mathrm{t}}$} & \multicolumn{1}{c}{$b_{\mathrm{t}}$} & $a_{\mathrm{s}}$ & $b_{\mathrm{s}}$ \\
\hline 5.1 & $0.49(5)$ & $0.50(7)$ & $0.93(1)$ & $0.74(2)$ & $0.11(4)$ & $0.069(4)$ \\
$5.15(\mathrm{~B})$ & $0.35(6)$ & $0.44(4)$ & $1.41(22)$ & $1.12(33)$ & $0.17(4)$ & $0.089(3)$ \\
$5.15(\mathrm{~S})$ & $0.21(3)$ & $0.44(2)$ & $2.41(22)$ & $1.48(45)$ & $0.19(3)$ & $0.053(1)$ \\
5.2 & $0.19(2)$ & $0.44(4)$ & $2.56(8)$ & $1.25(21)$ & $0.20(6)$ & $0.054(2)$ \\
5.3 & $0.094(46)$ & $0.41(4)$ & $2.94(20)$ & $1.84(20)$ & $0.23(7)$ & $0.033(3)$ \\
6.5 & $0.022(4)$ & $0.37(1)$ & $3.27(5)$ & $3.27(5)$ & $0.33(2)$ & $0.016(7)$ \\
\hline
\end{tabular}




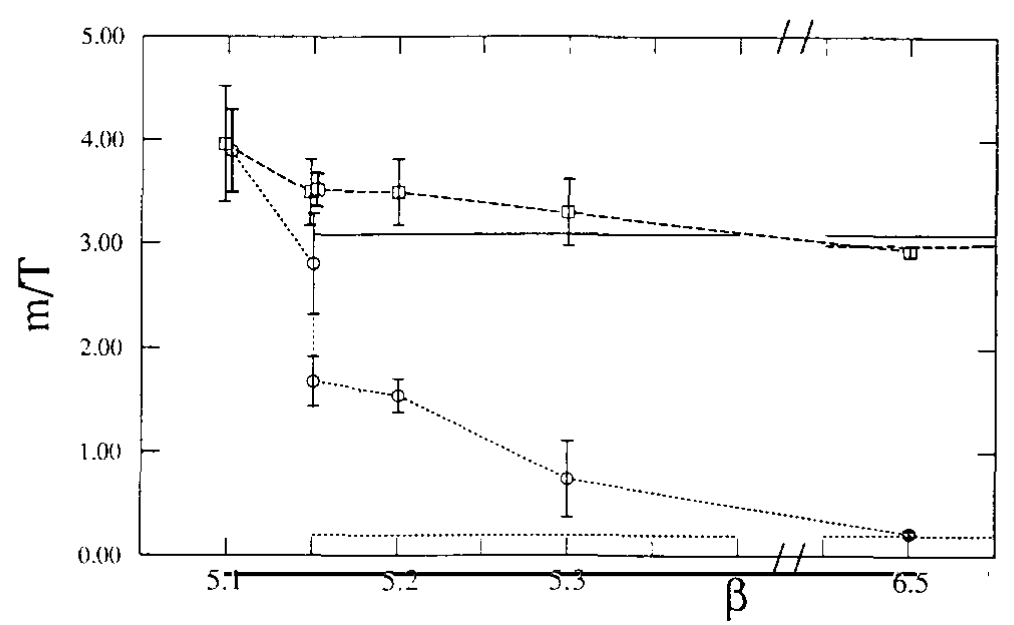

Fig. 5. The temporal (circles) and spatial (squares) effective quark masses in units of the temperature versus $\beta$. The solid line gives the $\left.O(g T)^{2}\right)$ result for the spatial screening mass, $m_{\mathrm{s}}$, calculated on a finite lattice with $g^{2}=1$, the dashed line below it indicates the corresponding free value $\left(g^{2}=0\right)$, $E_{\mathrm{s}, \min }$, defined in eq. (23), and the horizontal dotted line is the $\mathrm{O}\left((g T)^{2}\right)$ effective quark mass, $m_{\mathrm{t}}$, in lattice units on an $8 \times 16^{3}$ lattice taken from the fourth column of table 2 .

the strongly $\beta$-dependent effective quark mass is no longer important. Also the low-momentum part of the dispersion relation is consistent with the free fermion form, if $m_{\mathrm{t}}$ in eq. (28) is replaced by the measured zero-momentum values.

Modifications of the low-momentum part of the dispersion relation are expected to occur at finite temperature, due to interactions of quarks with the heat bath [12,13]. These are not visible in our simulation. However, this is not too surprising. In fact, deviations from the free particle dispersion relation are expected to be significant only for momenta much smaller than the temperature of

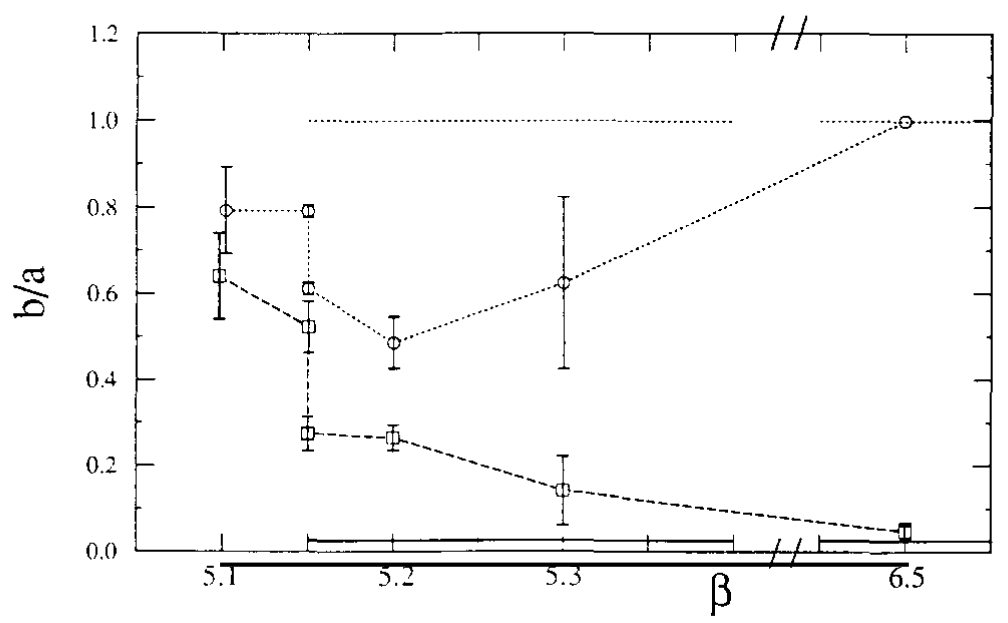

Fig. 6. Ratio of the amplitudes of the correlation function on even and odd sites of the lattice versus the gauge coupling $\beta$. Shown are results for temporal (circles) and spatial (squares) correlation functions. The horizontal lines correspond to the free field behaviour discussed in the text. Note that at $\beta=6.5$ the ratio $b_{\mathrm{t}} / a_{\mathrm{t}}$ has been set to unity in the fits to the correlation function (see table 3 ). 

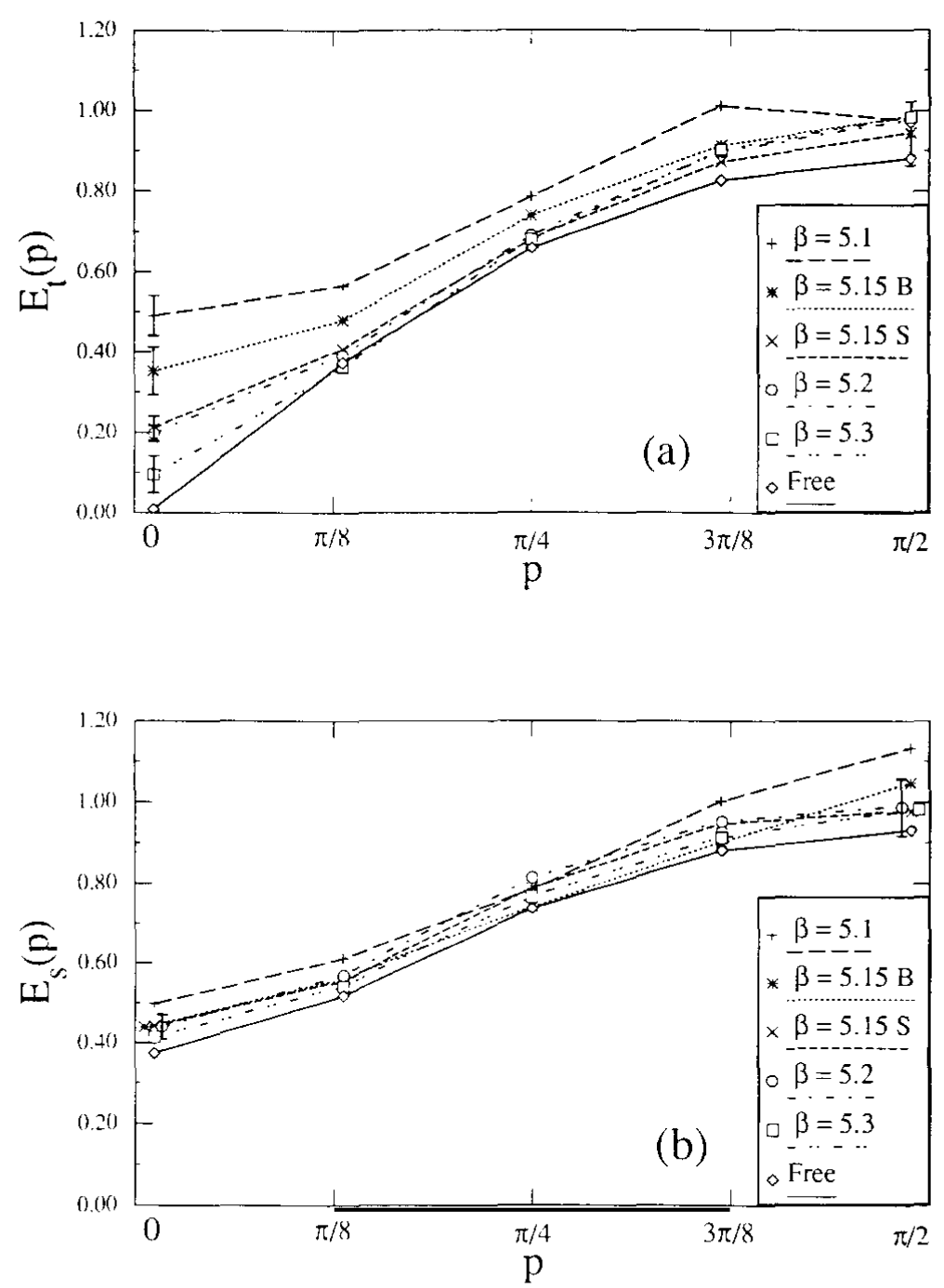

Fig. 7. Dispersion relations for temporal (a) and spatial (b) effective quark masses versus momentum $p=2 \pi n / 16, n=0,1,2,3$ and 4 , for various values of $\beta$ as given in the figure. For $p=0$ and $p=\pi / 2$ we show typical error bars. Data points have been displaced a bit for this purpose. Also shown are the free field dispersion relations (solid lines) using the corresponding free-field screening masses $m_{\mathrm{t}}=m$ (a) and $m_{\mathrm{s}}=\operatorname{arcsinh}\left(\sin (\pi / 8)^{2}+m^{2}\right)(\mathrm{b})$.

the system. An analysis of such effects will thus require much larger spatial lattices as our smallest non-zero momentum, $p_{1}=\pi / N_{\sigma}=\pi / 16$, is already of the order of the temperature of the system, $T=1 / N_{\tau}=1 / 8$.

\section{Conclusions}

We have studied the temperature dependence of the quark propagator on the lattice for four-flavour QCD with staggered fermions. The structure of the temporal as well as the spatial correlations functions does not show any strong deviations 
from free field behaviour in the chirally symmetric phase. In fact, for temperatures $T \gtrsim 1.75 T_{\mathrm{c}}$, the screening masses, dispersion relations and amplitudes of the correlation functions, which we have calculated all start showing perturbative behaviour. In particular, the sensitivity of the temporal correlation functions to the chiral phase transition and their rapid approach to perturbative predictions is remarkable. This suggests that a similar analysis of temporal meson and baryon correlation functions could yield important new information on the existence of bound states in these channels. In this case, however, the gluon exchange between quarks will be particularly important for the formation of bound states. It will be interesting, therefore, to analyze the modification of the hadronic correlation functions due to the corresponding $O\left(g^{2}\right)$ gluon exchange diagrams. Work in this direction is in progress.

At present the simulations with dynamical fermions are restricted to rather small lattices. The $8 \times 16^{3}$ lattice, used in this analysis, is about the smallest lattice, which can be used to measure temporal correlation functions and dispersion relations. In order to check details of the continuum perturbation theory, such as the existence of particle/hole excitations, and in particular the low-momentum structure of the quark dispersion relation, much larger lattices will have to be used in the future.

The numerical work described was performed on the CRAY-YMP at HLRZ, Jülich. Financial suppport from DFG under contract Pe 340/1-3 and the Ministerium für Wissenschaft und Forschung NRW under contract IVA5-10600990 is gratefully acknowledged.

\section{Appendix A}

Here we discuss the fermionic self-energy on a euclidean lattice of size $N_{\tau} \times N_{\sigma}^{3}$. We consider fermion fields with periodic boundary conditions imposed in the spatial directions and antiperiodic in the temporal. Then the fermion momenta take on the discrete values

$$
\begin{aligned}
& q_{0}=\frac{\pi}{N_{\tau}}\left(2 i_{0}+1\right), \quad i_{0}=0,1, \ldots, N_{\tau}-1 . \\
& q_{k}=\frac{2 \pi}{N_{\sigma}} i_{k}, \quad i_{k}=0,1, \ldots, N_{\sigma}-1, \quad(k=1,2,3) .
\end{aligned}
$$

The contribution from the first Feynman diagram in fig. 1 is given by

$$
\Sigma^{\mathrm{a}}\left(p_{0}, \boldsymbol{p}\right)=g^{2} C_{\mathrm{F}}\left[\sum_{k=0}^{3} i \gamma_{k} \tilde{\Sigma}_{k}^{\mathrm{a}}\left(p_{0}, \boldsymbol{p}\right)+m \tilde{\Sigma}_{m}\left(p_{0}, \boldsymbol{p}\right)\right],
$$


with $C_{\mathrm{F}}=\left(N^{2}-1\right) / 2 N$. The various terms are

$$
\begin{aligned}
\tilde{\Sigma}_{k}^{\mathrm{a}}= & -\frac{1}{N_{\tau} N_{\sigma}^{3}} \sum_{q_{0}} \sum_{q_{k}} \frac{\sin \left(q_{k}\right)}{4\left(\omega_{\mathrm{B}}^{2}+\sin ^{2}\left(\left(p_{0}-q_{0}\right) / 2\right)\right)\left(\omega_{\mathrm{F}}^{2}+\sin ^{2}\left(q_{0}\right)\right)} \\
& \times\left[\cos ^{2}\left(\frac{p_{k}+q_{k}}{2}\right)-\sum_{j \neq k} \cos ^{2}\left(\frac{p_{j}+q_{j}}{2}\right)\right], \\
\tilde{\Sigma}_{m}= & \frac{1}{N_{\tau} N_{\sigma}^{3}} \sum_{q_{0}} \sum_{q_{k}} \frac{1}{4\left(\omega_{\mathrm{B}}^{2}+\sin ^{2}\left(\left(p_{0}-q_{0}\right) / 2\right)\right)\left(\omega_{\mathrm{F}}^{2}+\sin ^{2}\left(q_{0}\right)\right)} \\
& \times \sum_{j=0}^{3} \cos ^{2}\left(\frac{p_{j}+q_{j}}{2}\right) .
\end{aligned}
$$

The second diagram in fig. 1 gives

$$
\begin{aligned}
\Sigma^{\mathrm{b}}\left(p_{0}, \boldsymbol{p}\right) & =g^{2} C_{\mathrm{F}} \sum_{k=0}^{3} i \gamma_{k} \tilde{\Sigma}_{k}^{\mathrm{b}}\left(p_{0}, \boldsymbol{p}\right) \\
\tilde{\Sigma}_{k}^{\mathrm{b}} & =-\sin \left(p_{k}\right) \frac{1}{N_{\tau} N_{\sigma}^{3}} \sum_{q_{0}} \sum_{q_{k}} \frac{1}{8\left(\omega_{\mathrm{B}}^{2}+\sin ^{2}\left(\left(p_{0}-q_{0}\right) / 2\right)\right)} .
\end{aligned}
$$

Here $\omega_{\mathrm{B}}$ and $\omega_{\mathrm{F}}$ are given by

$$
\begin{aligned}
& \omega_{\mathrm{B}}=\sqrt{\sum_{k=1}^{3} \sin ^{2}\left(\left(p_{k}-q_{k}\right) / 2\right)}, \\
& \omega_{\mathrm{F}}=\sqrt{\sum_{k=1}^{3} \sin ^{2}\left(q_{k}\right)+m^{2}} .
\end{aligned}
$$

We further define

$$
\begin{aligned}
& E_{\mathrm{B}}=2 \ln \left(\omega_{\mathrm{B}}+\sqrt{\omega_{\mathrm{B}}^{2}+1}\right), \\
& E_{\mathrm{F}}=\ln \left(\omega_{\mathrm{F}}+\sqrt{\omega_{\mathrm{F}}^{2}+1}\right) .
\end{aligned}
$$

In the infinite-volume limit, $N_{\sigma} \rightarrow \infty$, the sum over spatial momenta can be replaced by an integral

$$
\frac{1}{N_{\sigma}^{3}} \sum_{q_{1}, q_{2}, q_{3}} \rightarrow \frac{1}{(2 \pi)^{3}} \int \mathrm{d}^{3} \boldsymbol{q} .
$$


In the following we will use the notation $\int_{q}$ for either the finite lattice sums or the three-dimensional integral over spatial momenta. The sum over $q_{0}$ can be performed explicitly using the mixed propagator representation for the free fermion and boson propagators [11] appearing in (A.3) to (A.5). In the case of fermions these are given by the free fermion correlation functions (20) and (21) and similar expressions for bosons are easily obtained. With this we find

$$
\tilde{\Sigma_{0}^{\mathrm{a}}}\left(p_{0}, \boldsymbol{p}\right)=\sin \left(p_{0}\right) \int_{q} \frac{A_{0}}{8 \omega_{\mathrm{B}} \sqrt{\omega_{\mathrm{B}}^{2}+1} \sqrt{\omega_{\mathrm{F}}^{2}+1}}
$$

where the amplitude, $A_{0}$, is obtained after some lengthy but straightforward calculation as

$$
A_{k}=\left(1-n_{\mathrm{F}}+n_{\mathrm{B}}\right) f_{k}\left(x_{-}\right)+\left(n_{\mathrm{F}}+n_{\mathrm{B}}\right) f_{k}\left(x_{+}\right) \text {. }
$$

For $k=0$ the function $f_{0}(x)$ is defined as

$$
\begin{gathered}
f_{0}(x)=\frac{-1}{(1-x)^{2}+4 x \sin ^{2}\left(p_{0}\right)}\left[3 x \cosh \left(E_{\mathrm{F}}\right)\right. \\
-\frac{1}{2}\left(x^{2} \exp \left(E_{\mathrm{F}}\right)+\exp \left(-E_{\mathrm{F}}\right)\right)-4 x \sin ^{2}\left(p_{0}\right) \cosh \left(E_{\mathrm{F}}\right) \\
\left.+2 \sqrt{x}(1+x)\left(\frac{1}{2}-\sum_{k=1}^{3} \cos ^{2}\left(\left(p_{k}+q_{k}\right) / 2\right)\right)\right] \\
x_{-}=\exp \left(-2\left(E_{\mathrm{F}}+E_{\mathrm{B}}\right)\right), \\
x_{+}=\exp \left(-2\left(E_{\mathrm{F}}-E_{\mathrm{B}}\right)\right), \\
n_{\mathrm{F}}=\frac{1}{\mathrm{e}^{E_{\mathrm{F}} N_{\tau}}+1}, \\
n_{\mathrm{B}}=\frac{1}{\mathrm{e}^{E_{\mathrm{B}} N_{\tau}}-1} .
\end{gathered}
$$

We note that the structure of the amplitude, $A_{0}$, is very similar to the continuum result. In particular only the second term in (A.10) gives rise to the singular behaviour of the self-energy at small momentum $p_{0}$; in the continuum limit $x_{+} \rightarrow 1$ and $f_{0}$ thus becomes proportional to $1 / \sin ^{2}\left(p_{0}\right)$.

Similarly we obtain for $\tilde{\Sigma}_{k}^{\mathrm{a}}$ and $\tilde{\Sigma}_{m}^{\mathrm{a}}$ :

$$
\begin{aligned}
& \tilde{\Sigma}_{k}^{\mathrm{a}}\left(p_{0}, \boldsymbol{p}\right)=\int_{q} \frac{A_{k} \sin \left(q_{k}\right)}{8 \omega_{\mathrm{F}} \omega_{\mathrm{B}} \sqrt{\omega_{\mathrm{B}}^{2}+1} \sqrt{\omega_{\mathrm{F}}^{2}+1}} \quad(k=1,2,3), \\
& \tilde{\Sigma}_{m}^{\mathrm{a}}\left(p_{0}, \boldsymbol{p}\right)=\int_{q} \frac{A_{m}}{8 \omega_{\mathrm{F}} \omega_{\mathrm{B}} \sqrt{\omega_{\mathrm{B}}^{2}+1} \sqrt{\omega_{\mathrm{F}}^{2}+1}},
\end{aligned}
$$


where the amplitudes, $A_{k}$ and $A_{m}$, are given by (A.10) with

$$
\begin{aligned}
f_{k}(x)= & \frac{-1}{(1-x)^{2}+4 x \sin ^{2}\left(p_{0}\right)}\left[\frac{1}{2} \sqrt{x} \times\left(\left(\exp \left(E_{\mathrm{F}}\right)-x \exp \left(-E_{\mathrm{F}}\right)\right)\right.\right. \\
& \left.\times\left(1-2 \sin ^{2}\left(p_{0}\right)\right)-x \exp \left(E_{\mathrm{F}}\right)+\exp \left(-E_{\mathrm{F}}\right)\right) \\
& \left.+\left(1-x^{2}\right)\left(\frac{1}{2}+\sum_{0<i \neq k} \cos ^{2}\left(\left(p_{i}+q_{i}\right) / 2\right)-\cos ^{2}\left(\left(p_{k}+q_{k}\right) / 2\right)\right)\right] \\
f_{m}(x)= & \frac{1}{(1-x)^{2}+4 x \sin ^{2}\left(p_{0}\right)}\left[\frac{1}{2} \sqrt{x} \times\left(\left(\exp \left(E_{\mathrm{F}}\right)-x \exp \left(-E_{\mathrm{F}}\right)\right)\right.\right. \\
& \left.\times\left(1-2 \sin ^{2}\left(p_{0}\right)\right)-x \exp \left(E_{\mathrm{F}}\right)+\exp \left(-E_{\mathrm{F}}\right)\right) \\
& \left.+\left(1-x^{2}\right)\left(\frac{1}{2}+\sum_{i=1}^{3} \cos ^{2}\left(\left(p_{i}+q_{i}\right) / 2\right)\right)\right]
\end{aligned}
$$

\section{References}

[1] C. DeTar, Phys. Rev. D32 (1985) 276; D37 (1987) 2328

[2] E. Manousakis and J. Polonyi, Phys. Rev. Lett. 58 (1987) 847

[3] J. Engels, J. Fingberg, F. Karsch, D. Miller and M. Weber, Phys. Lett. B252 (1990) 625

[4] C. DeTar and J. Kogut, Phys. Rev. Lett. 59 (1987) 399; Phys. Rev. D36 (1987) 2828

[5] S. Gottlieb, W. Liu, D. Toussaint, R.L. Renken and R.L. Sugar, Phys. Rev. Lett. 59 (1987) 1881

[6] K. Born, S. Gupta, A. Irbäck, F. Karsch, E. Laermann, B. Petersson and H. Satz, Phys. Rev. Lett. 67 (1991) 302

[7] C. Bernard, M.C. Ogilvie, T. DeGrand, C. DeTar, S. Gottlieb, A. Krasnitz, R. Sugar and D. Toussaint, The spatial structure of screening propagators in hot QCD. University of Arizona preprint, AZPH-TH/91-60 (1991)

[8] A. Gocksch and A. Soni, Z. Phys. C53 (1992) 517

[9] G. Baym and N.D. Mermin, J. Math. Phys. 2 (1961) 232

[10] F. Karsch and H.W. Wyld, Phys. Rev. D35 (1987) 2518

[11] R.D. Pisarski, Nucl. Phys. B309 (1988) 476; Physica A158 (1989) 146; How to compute scattering amplitudes in hot gauge theories, Fermilab-PUB-88/113-T (November 1988), unpublished

[12] H.A. Weldon, Phys. Rev. D40 (1989) 2410

[13] G. Gatoff and J. Kapusta, Phys. Rev. D41 (1990) 611

[14] I. Bender, T. Hashimoto, F. Karsch, V. Linke, A. Nakamura, M. Schiestl and I.O. Stamatescu, Nucl. Phys. B (Proc. Suppl.) 17 (1990) 387; 20 (1991) 329

[15] V. Koch, E.V. Shuryak, G.E. Brown and A.D. Jackson, The propagation of quarks in the spatial direction in hot QCD, Stony Brook preprint SUNY-NTG-92-4

[16] V.V. Klimov, Sov. J. Nucl. Phys. 33 (1981) 934

[17] R.V. Gavai, S. Gupta, A. Irbäck, F. Karsch, S. Meyer, B. Petersson, H. Satz and H.W. Wyld, Phys. Lett. B241 (1990) 567

[18] F. Karsch, J.B. Kogut, D.K. Sinclair and H.W. Wyld, Phys. Lett. B188 (1987) 353

[19] R. Altmeyer, K.D. Born, M. Göckeler, R. Horsley, E. Laermann and G. Schierholz, HLRZ preprint HLRZ 92-17 (April 1992)

[20] P. van Baal, Nucl. Phys. B369 (1992) 259

[21] E. Laermann, R. Altmeyer, K.D. Born, M. Göckeler, R. Horsley, W. Ibes, T.F. Walsh and P.M. Zerwas, Nucl. Phys. B (Proc. Suppl.) 20 (1991) 380 